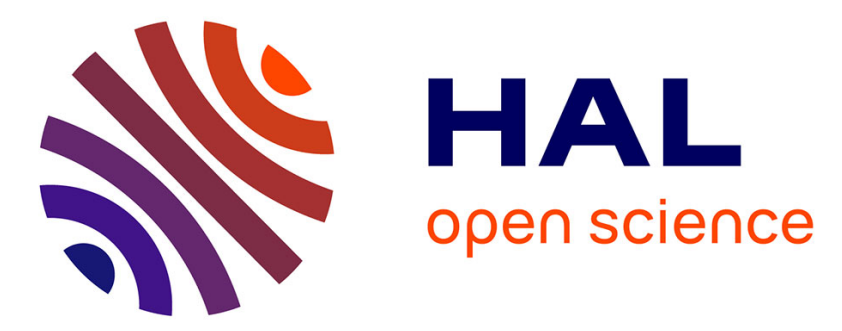

\title{
L'institutionnalisation d'un pouvoir judiciaire européen incertain en quête de légitimité : l'unité de coopération Eurojust.
}

Antoine Mégie

\section{- To cite this version:}

Antoine Mégie. L'institutionnalisation d'un pouvoir judiciaire européen incertain en quête de légitimité: l'unité de coopération Eurojust.. Politique européenne, 2007, numéro 23, pp.57-75. halshs00757144

\section{HAL Id: halshs-00757144 \\ https://shs.hal.science/halshs-00757144}

Submitted on 26 Nov 2012

HAL is a multi-disciplinary open access archive for the deposit and dissemination of scientific research documents, whether they are published or not. The documents may come from teaching and research institutions in France or abroad, or from public or private research centers.
L'archive ouverte pluridisciplinaire HAL, est destinée au dépôt et à la diffusion de documents scientifiques de niveau recherche, publiés ou non, émanant des établissements d'enseignement et de recherche français ou étrangers, des laboratoires publics ou privés. 


\title{
L'institutionnalisation d'un pouvoir judiciaire européen incertain en quête de légitimité : l'unité de coopération Eurojust.
}

\author{
Antoine Mégie \\ Université de Rouen
}

Depuis 2002, l'unité Eurojust constitue le premier office judiciaire européen en charge de favoriser la coopération entre les juridictions nationales des Etats membres de l'UE. Créée dans un contexte institutionnel et politique particulier, cette unité va avoir pour objectif principal de réussir à s'imposer comme un acteur incontournable de la coopération européenne en particulier vis-à-vis des autres instances nationales et supranationales compétentes. Cet article se propose d'analyser les logiques institutionnelles et sociales qui structurent le travail quotidien d'Eurojust dans cette quête de légitimité. L'étude des ressources mobilisées par les membres judiciaires de cette unité et les contraintes qui pèsent sur leur travail, nous permet de comprendre les raisons et la façon dont ces derniers tendent à retraduire le cadre formel et ainsi à redéfinir l'action d'Eurojust dans l'espace européen de sécurité.

Le 15 et 16 Octobre 1999 à Tampere, le Conseil européen décide, afin de favoriser la mise en œuvre «d'un espace de liberté, de sécurité et de justice dans l'Union européenne» (Conseil européen de Tampere, 1999), de créer la première unité judiciaire à l'échelon européen : Eurojust. Regroupant un représentant de l'administration judiciaire de chaque Etatmembre, cette unité européenne est chargée de renforcer la coordination multilatérale entre les autorités nationales. Le dispositif d'Eurojust marque une réelle évolution institutionnelle au regard des premiers systèmes d'entraide exclusivement horizontaux et bilatéraux que sont les magistrats de liaison et le Réseau judiciaire européen ${ }^{1}$.

\footnotetext{
${ }^{1}$ Crées respectivement en 1993 et 1998 , les dispositifs de magistrat de liaison et du Réseau judiciaire européen (RJE) ont pour objectif principal de favoriser l'échange d'informations entre les Etats membres en matière judiciaire, à travers la nomination à l'étranger (magistrats de liaison) ou au sein des juridictions nationales (RJE) de juges en charge des questions d'entraide européenne. Ces derniers n'ont pour autant aucun pouvoir spécifique. La mise en place de ces structures s'inscrit dans une approche bilatérale et temporaire de l'entraide
} 
La mise en oeuvre de cette institution judiciaire européenne concrétise pour une part le mouvement d'européanisation qui depuis le traité de Maastricht et d'Amsterdam, touche le domaine de la sécurité intérieure (Bigo, 1996). Largement dominé par les autorités nationales via le Conseil européen, le secteur intergouvernemental de la coopération en matière pénale s'européanise à travers des relations institutionnelles oscillant entre compétition et collaboration (Mégie, Ravinet, 2007, p 87-143). La décision de mettre en place l'unité Eurojust lors du sommet européen de Tampere illustre parfaitement cette configuration.

En réaction aux diverses propositions présentées par la Commission européenne, en particulier le projet Corpus Juris qui prévoyait la mise en place du principe de territorialité européenne à travers l'établissement d'un Procureur européen ${ }^{2}$, différents Etats (Belgique, France, Portugal et Suède) décident, avec l'aide du Secrétariat général du Conseil, de soutenir le projet d'une unité judiciaire de type intergouvernemental. Aboutissement de ce projet des « quatre présidences », Eurojust représente ainsi la réponse construite par les Etats face à la volonté de la Commission européenne de communautariser une partie du domaine judiciaire (Mangenot, Michel, Paris, 2005 ; Mégie, 2006).

Le 10 décembre 2002, le collège d'Eurojust ${ }^{3}$ débute ses activités dans les locaux de La Haye. Eurojust devient la troisième unité à l'échelle européenne en charge des questions de coopération pénale aux côtés de l'unité Europol (office de Police) et de l'OLAF (Office communautaire de lutte anti-fraude).

Dans un secteur européen encore en cours d'institutionnalisation, qui se caractérise par une superposition des règles, une concurrence des dispositifs et une forte incertitude juridique (Domenach, 2006, p 149-168), l'objectif principal d'Eurojust va être de réussir à s'imposer comme un acteur légitime par rapport aux autres autorités nationales et européennes

pénale, laissant à chaque Etat-membre le choix d'adopter (magistrat de liaison) ou d'organiser (RJE) ces dispositifs en fonction de leur organisation judiciaire intérieure.

${ }^{2}$ Depuis le début des années 1990, la question de la protection du budget communautaire est devenue une problématique centrale du domaine de la sécurité auquel se rattache un ensemble de projets, pilotés par la DG Justice, Liberté et Sécurité (JLS), en faveur d'une plus grande intégration pénale. Dans ces conditions, l'OLAF (Office de lutte anti-fraude), en charge de lutter contre les fraudes aux intérêts financiers de la Communauté européenne, va servir de point d'ancrage à cette stratégie de communautarisation en particulier à travers le projet Corpus Juris proposant la mise en place d'un Procureur européen. Ce positionnement correspond à une véritable volonté politique, de la part de la Commission, de s'imposer dans les négociations intergouvernementales en matière de coopération pénale, comme le souligne ce fonctionnaire européen : «à travers la dimension judiciaire du détournement des subventions européenne, nous avons voulu, tout en poussant les Etats à avancer, nous imposer dans les négociations afin de promouvoir une meilleure harmonisation». (Entretien réalisé avec un membre de la commission européenne, février 2003).

${ }^{3}$ Comme nous le verrons plus précisément par la suite, le collège, qui réunit l'ensemble des magistrats nationaux (25 personnes), représente l'organe central de l'unité Eurojust. Se réunissant une fois par semaine, avec pour condition un quorum des $2 / 3$, le collège d'Eurojust est une enceinte collégiale, dans laquelle chaque représentant dispose d'une voix. Le vote concernant le choix des affaires judiciaires traitées et des orientations institutionnelles se fait généralement à main levée à la majorité des deux tiers. 
compétentes. Cette volonté de trouver une position institutionnelle et judiciaire stable influence fortement l'activité des magistrats de l'unité. A travers leurs pratiques quotidiennes ces derniers vont retraduire le cadre formel instauré par la décision-cadre du 28 février 2002 et faire ainsi évoluer l'institution.

En inscrivant, d'un point de vue théorique, notre réflexion sur le changement dans les hypothèses néo-institutionnelles, nous tenterons de fournir un cadre d'interprétation aux orientations prises par Eurojust. L'analyse des ressources, des représentations et des relations interpersonnelles des membres de cette unité permet, dans ce contexte, de questionner concrètement les mécanismes d'ajustement. La prise en compte du phénomène de path dependence (Pierson, 1993), nous conduit à comprendre comment et jusqu'où le cadre institutionnel pèse sur les comportements et sur les représentations de ces acteurs. Par ailleurs, le fait de centrer notre étude sur les pratiques des acteurs nous amène à combiner cette première perspective avec les problématiques centrales des approches de sociologie des organisations. En effet, mesurer les évolutions d'Eurojust à travers ce prisme, nous renvoie à la manière dont les acteurs, inscrits dans de multiples contraintes, cherchent à s'en affranchir en puisant des ressources dans les réglementations, les structures formelles ou les objets techniques (Musselin, 2005, p 58).

Cet article se propose donc, en s'appuyant sur la littérature officielle ${ }^{4}$ et sur une série d'entretiens $^{5}$, d'expliquer les logiques institutionnelles et sociales qui structure le travail d'Eurojust dans sa volonté de s'imposer en tant qu'instance légitime de la coopération européenne en matière de sécurité. Nous verrons à travers l'étude des usages quotidiens des magistrats ${ }^{6}$ de cette unité quelles sont les contraintes et ressources qui influent sur un tel positionnement institutionnel. Le choix de mettre en exergue ces dynamiques est d'autant plus pertinent que la compréhension des logiques qui guident Eurojust dans sa recherche de légitimité conduit à appréhender plus largement l'orientation actuelle du domaine européen de « justice, liberté et sécurité ».

\footnotetext{
${ }^{4}$ En particulier les rapports d'activités d'Eurojust. Il s'agit en l'espèce des rapports d'activités de l'unité Eurojust actuellement publiés, c'est-à-dire de 2002 à 2005. Disponibles à l'adresse : http://www.eurojust.europa.eu/press_annual.htm.

${ }^{5}$ Ces entretiens ont été réalisés entre $20 \overline{0} 1$ et 2006 avec des magistrats membres d'Eurojust mais également avec des magistrats participant aux négociations européennes. Nous avons également effectué des interviews avec des juges n'ayant aucun poste européen afin d'interroger la manière dont l'échelon européen est intégré dans le travail quotidien des autorités judiciaires nationales. Afin de respecter l'anonymat - souvent demandé - des personnes interviewées, nous ne donnerons, dans la majorité des cas, que la nationalité et/ou la fonction des personnes citées.

${ }^{6}$ Dans un souci de compréhension et de simplicité, et malgré la diversité des systèmes judiciaires de chaque Etat membre, nous utiliserons le terme de magistrat et/ou de procureur afin de qualifier les membres judiciaires d'Eurojust.
} 
L'analyse des relations interinstitutionnelles qui existent entre Eurojust et les autres autorités compétentes nous permettra, en premier lieu, de comprendre la configuration de l'espace européen de sécurité et la place de l'unité judiciaire. Nous, nous intéresserons plus précisément aux rapports qu'Eurojust entretient avec les autorités judiciaires nationales et les offices européens que sont Europol et l'OLAF. A partir de cette présentation, il conviendra, dans un second temps, d'interroger les pratiques des magistrats d'Eurojust afin de rendre compte des logiques concurrentes et complémentaires à l'origine de la redéfinition institutionnelle et opérationnelle de l'unité. L'analyse des ressources mobilisées par ces acteurs et des contraintes qui pèsent sur leur travail, nous permettra de comprendre les raisons et la façon dont Eurojust oriente actuellement son action judiciaire dans sa quête de légitimité.

\section{La position institutionnelle de l'unité Eurojust dans l'espace européen de sécurité : entre instabilité et défiance.}

Etudier la place actuelle d'Eurojust dans l'espace européen de sécurité renvient à analyser les relations interinstitutionnelles à travers leur dimension juridique mais aussi via leur fonctionnement pratique. Nous verrons alors comment la hiérarchie institutionnelle vis-àvis des autorités nationales, et l'absence de cadre juridique de coopération avec les autres unités européennes, doivent être mises en perspective avec les usages quotidiens des magistrats d'Eurojust.

\subsection{Des relations incertaines entre Eurojust et les autorités nationales}

Constituant une agence dotée de la personnalité juridique, la principale caractéristique de l'unité Eurojust réside dans sa dimension intergouvernementale. Opposés au projet de la Commission d'un ministère public européen, les Etats vont définir Eurojust comme une unité dépendante des autorités nationales. Au niveau institutionnel, cette orientation politique a pour conséquence de faire des procureurs siégeant à Eurojust des «membres nationaux », sans prérogatives spécifiques et ancrés dans le dispositif judiciaire de leur pays d'origine, à la différence du personnel administratif qui est assimilé, pour son recrutement et son statut, aux fonctionnaires des Communautés européennes ${ }^{7}$. Dans cette logique, l'organigramme de

\footnotetext{
${ }^{7}$ En raison de ce statut d'agent communautaire, le personnel administratif est directement dépendant du collège et n'est pas sous l'autorité des gouvernements ou autres autorités extérieures à Eurojust (Décision instituant Eurojust, 2002, article 29 et 30). Dans le cadre de cet article nous ne prendrons pas en compte l'administration d'Eurojust comme objet d'étude. Pour autant, son analyse reste essentielle dans la compréhension plus générale de l'évolution institutionnelle de l'unité.
} 
l'office se structure autour du collège qui, regroupant l'ensemble des membres nationaux, constitue l'enceinte décisionnelle principale ${ }^{8}$.

\section{Organigramme : (source Eurojust)}

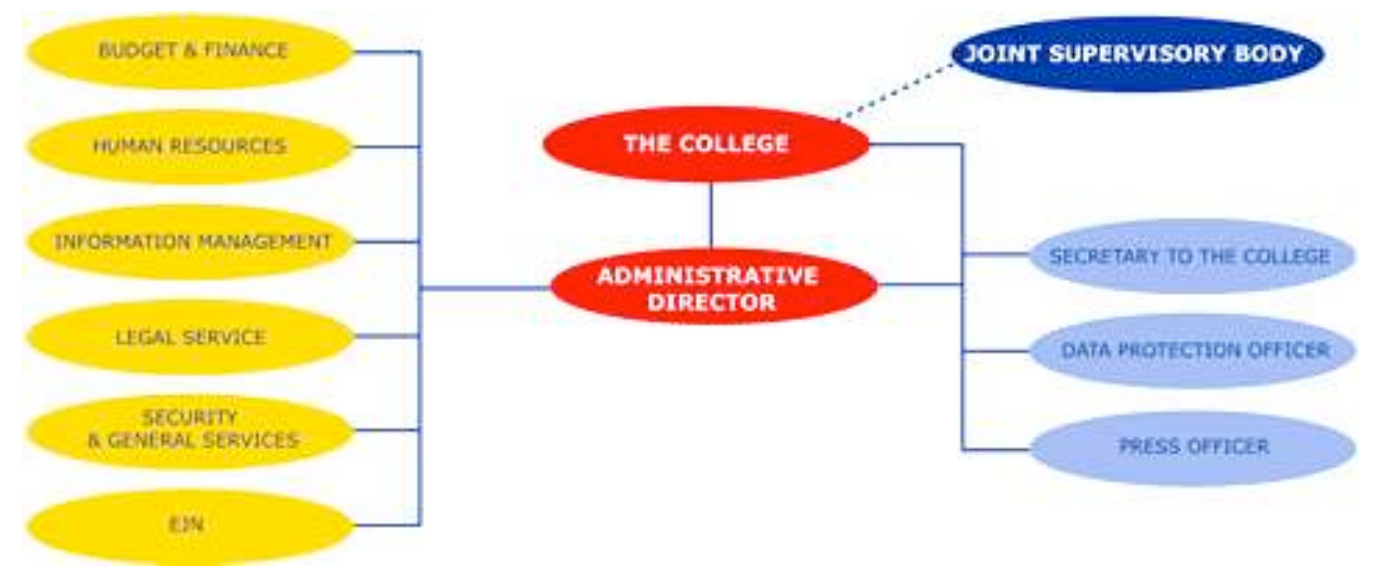

Les autorités nationales sont les seules à pouvoir saisir et déclencher, de manière contraignante, une enquête pénale transnationale ${ }^{9}$. Dans ces conditions, l'unité européenne apparaît, avant tout, comme «un prestataire de service » pour les autorités nationales. Les relations avec les juridictions nationales ont donc un impact direct sur le volume d'affaires traitées par Eurojust et sur la possibilité pour cette unité de s'imposer comme un maillon essentiel de la coopération judiciaire. De l'aveu même de ses membres, Eurojust ne peut se pérenniser et donner des résultats que si les magistrats nationaux s'en saisissent et y recourent.

Or, malgré une progression constante des affaires traitées depuis 2002, le constat fait par l'unité elle-même est relativement pessimiste : «certains Etats membres confient moins de dossiers qu'on pourrait en attendre et, même dans les juridictions qui recourent régulièrement à Eurojust, de nombreuses affaires ont été traitées sans sa coopération bien que celle-ci eut apporté, sans l'ombre d'un doute, une valeur ajoutée » (Rapport Eurojust, 2005, p 68). De manière générale, et au-delà du discours officiel, les relations entre Eurojust et les autorités nationales semblent être dominées par une très forte incertitude due en particulier à une résistance de la part des magistrats nationaux. De nos entretiens effectués avec des juges français, belges, néerlandais et italiens exerçant leur pouvoir à l'échelle

\footnotetext{
${ }^{8}$ A côté des décisions concernant l'organisation interne, le principal travail du collège est de décider des affaires judiciaire qu'Eurojust doit traiter.

${ }_{9}^{9}$ Les pouvoirs judiciaires dévolus aux membres d'Eurojust seront étudiés plus précisément dans la seconde partie de cet article.
} 
nationale $^{10}$, il résulte que la majorité d'entre eux se considèrent «éloignés» des problématiques européennes : «toutes les affaires que nous traitons ne sont pas à dimension européenne. Concrètement, nous n'avons que rarement des affaires transnationales ${ }^{11}$. Parallèlement à cette première explication relative à la nature des infractions, d'autres facteurs expliquent les résistances rencontrées au niveau national.

Le premier réside sans aucun doute dans le savoir qu'il soit juridique ou linguistique. Ces enjeux de connaissances ne concernent pas uniquement le cas de l'unité Eurojust mais intéressent plus généralement l'ensemble du domaine de la coopération européenne en matière de sécurité. En étroite relation avec ces questions, les facteurs structurels apparaissent également déterminants. Pour les juges de l'échelon national, le niveau européen est souvent considéré comme complexe. Dans le cas de la France, en raison de ces représentations, les juges hésitent souvent à mobiliser les instruments de coopération comme Eurojust, car ceux-ci ne répondent pas, selon eux, à leurs objectifs d'efficacité imposés par un système national caractérisé, de plus en plus, par une productivité rationalisée (Bastard, Mouhanna, Ackermann, 2005). Comme le souligne un magistrat, "si l'on a cent dossiers dans son cabinet, il faut traiter les dossiers rapidement, ce que la prise en compte de la dimension européenne ne permet pas ${ }^{12}$. Un autre juge résume la tendance qui s'impose au sein des cabinets judiciaires nationaux : "prendre en compte la dimension européenne des affaires demande des compétences et du temps. Il faut un investissement pas toujours évident eu égard au nombre de dossiers à traiter et aux moyens qui nous sont donnés ${ }^{13}$. Enfin, les pratiques et usages judiciaires peuvent aussi expliquer certains refus : «les magistrats sont toujours attachés à leur indépendance. La majorité d'entre nous a l'habitude de traiter seul ses affaires $»^{14}$. Le partage d'une instruction avec un autre magistrat de surcroît étranger est souvent assimilé à une perte de pouvoir et d'autorité sur la conduite de la procédure.

Face à ces multiples résistances, Eurojust va focaliser une grande partie de son action en faveur d'un renforcement des liens avec les autorités judiciaires nationales. Participant à sa stratégie de légitimation, cette démarche représente une part essentielle de l'activité des membres de 1'unité depuis 2002, «on prend notre bâton de pèlerin et on essaye de faire connaître l'unité à tous les échelons, que se soit dans les juridictions mais aussi dans le cadre

\footnotetext{
${ }^{10}$ Réalisés, dans le cadre d'un travail de thèse, l'objectif de tels interviews est de saisir la manière dont le travail de l'unité Eurojust, et plus largement des autres dispositifs de coopération, est perçu par les juges du niveau national qui ne sont pas spécialisés dans ce type de contentieux.

${ }^{11}$ Entretien réalisé avec un magistrat français, février 2003.

${ }^{12}$ Entretien réalisé avec un magistrat français, juin 2005.

${ }^{13}$ Entretien réalisé avec un magistrat néerlandais, février 2003.

${ }^{14}$ Entretien réalisé avec un magistrat d'Eurojust, février 2004.
} 
des formations ou des écoles de magistrats $»^{15}$. L'objectif est ainsi de « sensibiliser les juges sur notre unité et de leur montrer que nous sommes efficaces. Mais il faut du temps et surtout des résultats ${ }^{16}$. Les points de résistances que nous avons soulignés précédemment se retrouvent au centre du discours des membres d'Eurojust : " nous devons expliquer tous les avantages. Dire que nous pouvons les aider si ils ne connaissent pas très bien le droit de l'autre pays, si ils ne parlent pas la langue. Nous devons aussi leur dire que nous pouvons faciliter leur travail et ainsi leur faire gagner du temps etc. ${ }^{17}$. Ce travail de sensibilisation est encore à l'heure actuelle l'un des principaux objectifs de l'unité.

Si les résistances se situent essentiellement du côté des autorités nationales, il est important de noter que les membres d'Eurojust ne s'inscrivent que très peu dans une logique d'autonomisation vis-à-vis de l'échelon national. L'utilisation extrêmement rare des articles 6 et 7 de la décision-cadre de 2002, permettant d'émettre une requête officielle à l'encontre des autorités nationales non disposées à soumettre un dossier, en apporte la preuve. Entre 2002 et 2005, seulement deux saisies de ce type ont été réalisées. Les propos officiels du collège sur ce point sont révélateurs : «les membres nationaux sont réticents à agir contre le gré des autorités nationales. Ils ressentent comme une perte de prestige d'avoir à présenter une requête officielle, conformément à l'article 6 et 7 (...). Par ailleurs, dans une certaine mesure, les membres nationaux se sentent et agissent davantage comme des ambassadeurs de leurs autorités nationales que comme des partisans de l'idée d'une coopération transfrontalière dans les affaires criminelles » (Rapport Eurojust, 2005, p 111). En réalité, il semble que, malgré la dimension non contraignante de ce type de demande, les membres nationaux hésitent à utiliser une telle procédure afin de ne pas créer de conflits avec les autorités nationales de leur propre pays qui peuvent considérer ce recourt comme une atteinte à leur indépendance. En d'autres termes, pour les membres d'Eurojust, l'emploi constant de telles prérogatives peut devenir un obstacle à leur travail de sensibilisation en direction des confrères du niveau national. La voie informelle et personnelle est, dans ce contexte, très largement privilégiée afin d'établir des relations de confiance.

Enfin, dans certains cas, si la coopération entre Eurojust et les juges nationaux fonctionne, l'incertitude reste néanmoins encore présente en matière d'équilibre juridique. De par ses prérogatives, l'unité européenne peut intervenir dans certaines procédures afin de

\footnotetext{
${ }^{15}$ Ibid.

${ }^{16}$ Entretien réalisé avec un membre d’Eurojust, mai 2001.

${ }^{17}$ Entretien réalisé avec un membre d'Eurojust, février 2003.
} 
régler des conflits de juridictions entre autorités nationales. Dans ces dossiers, il semble que les logiques qui dominent s'inscrivent avant tout dans la recherche d'une efficacité maximum permettant de renforcer son positionnement. Ces affaires sont, en effet, centrales pour Eurojust puisqu'elles lui permettent de montrer son «utilité » et donc sa légitimité sur des dossiers aussi sensibles que le terrorisme ou la réparation des dommages de la marée noire de l'Erika. On assiste ainsi à des processus de «jurisdiction shopping », au sens de «sélection d'une juridiction », guidés par une logique d'efficacité clairement revendiquée. Or, eu égard à l'absence d'harmonisation dans la qualification des infractions et des peines à l'échelle européenne, cela peut aboutir, sur des sujets aussi sensibles que le terrorisme, l'euthanasie, le trafic de drogue, le racisme à des incertitudes juridiques contraires, par définition, à l'équilibre et à la stabilité du droit que prévoit tout régime pénal. En effet, du fait de la grande diversité des systèmes pénaux européens, le choix de la juridiction et donc de l'Etat peut avoir des conséquences importantes sur la manière dont l'infraction va être qualifiée et sur les modalités de poursuite et de jugement de la personne concernée.

Les relations entre Eurojust et les juridictions nationales se caractérisent donc par une forte incertitude sur le plan opérationnel et juridique. Dans ces conditions, la recherche de légitimité vis-à-vis des magistrats de l'échelon national apparaît comme l'enjeu central pour l'unité. En raison de pouvoirs judiciaires limités, les membres d'Eurojust vont utiliser d'autres ressources afin de s'imposer comme des interlocuteurs « crédibles » et « incontournables ». Avant de s'intéresser directement à cette question, il est essentiel de prendre en compte les relations interinstitutionnelles à l'échelle européenne afin de terminer d'interroger la place d'Eurojust dans l'espace européen de sécurité.

\subsection{Une configuration interinstitutionnelle dominée par des logiques de compétitions.}

Comme nous l'avons souligné en introduction, l'OLAF et Europol constituent, aux côtés d'Eurojust, les deux autres unités européennes qui de par leur champ de compétences interviennent dans le domaine des affaires pénales. A l'instar des relations entretenues par l'unité judiciaire avec les autorités nationales, les rapports entre Eurojust et ces deux offices semblent être également marqués par une forte instabilité.

Le fait que les unités Eurojust et l'OLAF se soient construites sur des principes antagonistes, dans une logique de compétition institutionnelle, a des conséquences sur la façon dont ces deux offices se positionnent l'un par rapport à l'autre. Par rapport à l'architecture européenne, Eurojust traite des affaires concernant le troisième pilier, alors que 
l'OLAF n'est compétente que pour les affaires du premier pilier. Néanmoins, cette distinction institutionnelle n'est pas aussi claire sur le terrain des infractions. Au-delà des dispositions juridiques et politiques prônant une étroite collaboration ${ }^{18}$, Eurojust et l'OLAF semblent avoir des difficultés à coopérer et restent attachées à leur autonomie. D’ailleurs, le mémorandum, signé entre les deux parties, laisse une grande marge de manœuvre à chaque institution concernant le partage de l'information et la collaboration pratique : « en fait, cet accord est vide de sens ${ }^{19}$. Dans le jeu institutionnel européen, Eurojust et l'OLAF cherchent essentiellement à s'imposer afin d'asseoir leur légitimité en tant qu'acteur central de la coopération judiciaire. Crée en 1999, à la suite du scandale de la Commission Santer, l'OLAF a développé une stratégie affirmée en faveur d'une judiciarisation de son travail. Cette prise de position se traduira en 2000, juste après la création officielle d'Eurojust, par la mise en place d'une unité de magistrats communautaires en son sein. Cette évolution institutionnelle s'appuie en partie sur les orientations du projet Corpus Juris (Pujas, 2006).

L'établissement au sein de chaque unité de bureaux chargés du développement de cette collaboration n'a pas encore pu modifier ces logiques de compétition. A ce titre, les chiffres fournis dans le rapport d'activité d'Eurojust pour l'année 2005 sont intéressants. Eurojust a reçu, à travers les réunions de coopération bimensuelles, 11 cas de la part de l'OLAF. Sur ces 11 cas, 7 d'entres eux ont cependant été transmis avec des délais de coopération trop justes qui n'ont pas permis aux membres d'Eurojust de réellement intervenir.

Dans une logique similaire, la collaboration réelle entre l'unité Europol et Eurojust reste, elle aussi, très relative et instable. Lors de la négociation de la décision-cadre Eurojust, les Etats furent confrontés à la difficulté de ne pouvoir évoquer que les seuls devoirs d'Eurojust à l'égard d'Europol, en raison de l'obligation de rouvrir les négociations pour toute réforme de la convention Europol (voir Den Boer et Bruggeman dans ce numéro). Cette construction a alors fait craindre la subordination de l'entité judiciaire à l'organe de coopération policière (Rapport de l'Assemblée Nationale, 2002). Dans le même temps, l'une des principales justifications fournies par les promoteurs d'un Procureur européen résidait dans l'idée que face à l'institutionnalisation de plus en plus affirmée de la coopération policière via Europol, un encadrement judiciaire devait être imposé. Si cet objectif fut au cœur des revendications des magistrats et de leurs représentants syndicaux, il n'a eu en réalité aucun prolongement concret. Comme le confirme différents témoignages, cette piste n'a

\footnotetext{
${ }^{18}$ Voir en plus des nombreuses déclarations d'intention de la commission européenne et d'Eurojust, le mémorandum du 14 avril 2004 établissant les relations entre Eurojust et OLAF.

${ }^{19}$ Entretien réalisé avec un fonctionnaire européen, membre de l'OLAF, mars 2004.
} 
jamais été réellement jugée réaliste pour un grand nombre des membres d'Eurojust et a été ainsi très vite abandonnée.

Malgré les dispositions juridiques en faveur d'un renforcement de la collaboration (Accord entre Eurojust et Europol, 2004), les relations entre Eurojust et Europol restent distantes. Comme le confie un membre d'Eurojust concernant cet accord : "Chacun est resté sur ses positions en voulant garder ses pouvoirs et son terrain de compétences. Cet accord $n$ 'engage en rien» ${ }^{20}$. Les statistiques relatives aux échanges d'informations depuis 2004 entre les deux offices illustrent cet état de fait. En 2004, aucune demande n'a été émise par Europol auprès d'Eurojust. En 2005, Europol a émis une demande et a reçu une réponse. Dans le sens inverse, l'unité Eurojust a émis 8 demandes et a reçu 4 réponses en 2004. Pour l'année 2005, Eurojust a émis 64 demandes et a reçu 52 réponses. On constate qu'Eurojust sollicite plus la collaboration d'Europol, ce qui peut s'expliquer par la place centrale de cet office de police dans le recueil d'informations.

L'absence d'une réelle coopération entre Eurojust et les autres unités de l'espace européen de sécurité s'inscrit dans des dynamiques de compétition qui résultent de la superposition de compétences entre ces offices et de l'instabilité d'un champ européen de la sécurité en pleine institutionnalisation. La compréhension de la place incertaine d'Eurojust par rapport aux autres autorités compétentes permet de définir les facteurs exogènes qui pèsent sur le fonctionnement et les usages au sein de cette unité.

\section{Ajustements et redéfinitions des pouvoirs et des compétences judiciaires d'Eurojust}

Dans un environnement institutionnel instable et compétitif, les magistrats d'Eurojust tendent à redéfinir, par leurs pratiques, le cadre formel de l'institution afin de stabiliser et de légitimer leur action. L'analyse des pouvoirs judiciaires et du champ de compétences, nous permet de mettre en exergue un tel processus d'ajustement, et de comprendre les effets que celui-ci peut avoir sur l'orientation générale de cet office judicaire européen.

\subsection{L'utilisation des ressources personnelles comme moyen de contourner les contraintes}

\section{d'une organisation asymétrique}

En raison des dynamiques politiques et institutionnelles qui ont structuré l'adoption du dispositif Eurojust, nous avons déjà souligné la très forte dépendance du collège et de ses membres aux autorités nationales. Ainsi, si le personnel administratif est pris en charge par le

\footnotetext{
${ }^{20}$ Entretien réalisé avec un membre d'Eurojust, mars 2004.
} 
budget propre de l'unité, il n'en est pas de même pour les magistrats siégeant au collège. En effet, chaque Etat membre définit et paye le salaire de ses représentants. Au-delà de la simple rémunération personnelle, ce financement par les Etats a une incidence directe sur la composition des équipes nationales et donc sur le fonctionnement quotidien de l'unité.

La grande majorité des membres nationaux sont les seuls représentants de leur Etat au sein d'Eurojust. En 2005, huit pays avait nommé en plus de leurs représentants, un magistrat adjoint : Allemagne, France, Italie, Pays-bas, Portugal, Finlande, Suède et Royaume-Uni. Ces magistrats ont notamment le pouvoir de remplacer le représentant national lors des réunions du collège. Parmi ces huit pays, seuls trois Etats ont parallèlement désigné un assistant : Allemagne, France, Pays-Bas. Dans la pratique, cette situation crée des déséquilibres entre les différentes représentations nationales : «selon que vous avez des adjoints et assistants, cela change la charge de travail. Notre équipe est bien fournie, donc on peut mieux répartir le travail. Ce n'est pas le cas pour tous les autres représentants nationaux qui dans ce cas doivent tout faire $»^{21}$.

La dépendance vis-à-vis des autorités nationales dans les ressources financières et humaines de chaque magistrat d'Eurojust se prolonge sur le terrain des compétences judiciaires. Les Etats restent responsables du pouvoir qu'ils donnent à leurs représentants. La réalisation de l'unité Eurojust à l'échelon européen ne signifie donc pas un transfert de compétence judiciaire à ce niveau : «Chaque Etat membre définit la nature et l'étendue des pouvoirs judiciaires qu'il confère à son membre national sur son propre territoire. Il définit également le droit pour un membre national d'agir à l'égard des autorités judiciaires étrangères (...)» (Décision instituant Eurojust, 2002, article 9). L'intergouvernementalité du dispositif est par ailleurs largement affirmée à travers la possibilité pour chaque Etat de déterminer la durée du mandat de son représentant et de faire évoluer l'étendu des pouvoirs de ce dernier.

Dans la totalité des cas, les membres nationaux n'ont pas de contrôle direct sur les poursuites et enquêtes des autorités judiciaires nationales. Certains n'ont, par ailleurs, pas la possibilité de superviser et d'intervenir dans la phase préparatoire des enquêtes. Ainsi, si une partie des magistrats du collège d'Eurojust a la compétence de déclencher des poursuites ou d'émettre des commissions rogatoires internationales, beaucoup d'autres ne peuvent que communiquer les demandes et n'ont aucun pouvoir décisionnel. Une telle asymétrie conduit à un fort déséquilibre dans l'activité d'Eurojust selon les pays : «pour prendre un exemple qui

\footnotetext{
${ }^{21}$ Entretien réalisé avec un magistrat d'Eurojust, mars 2004.
} 
me concerne, je peux demander directement à un juge d'instruction de me donner des renseignements. Je peux regarder avec lui comment je peux lui apporter mon concours et cela se fait de la manière la plus directe et la plus opérationnelle (...). J'ai ce pouvoir, mais d'autres collègues sont beaucoup plus limités dans leurs compétences. Nous ne sommes pas tous au même niveau, cela peut poser des problèmes de droit $»^{22}$.

La question des prérogatives judiciaires et de leur asymétrie est systématiquement mise en avant, par les membres d'Eurojust, comme un des obstacles principaux au développement de leur efficacité et de facto de leur légitimité : «dans certains cas, on sait par avance qu'en raison des pouvoirs de nos collègues d'Eurojust, la coordination va être difficile. Donc on essaye de se renseigner en amont des réunions afin que la coordination ne débouche pas sur un échec qui pourrait discréditer notre action ${ }^{23}$. Du fait de l'hétérogénéité des pouvoirs dévolus au membre du collège, certains dossiers peuvent être traités de manière informelle, sans passer par le collège, voire écarter et orienter vers d'autres voix d'entraide.

A partir des témoignages recueillis et des rapports officiels, il semble que le manque de pouvoirs judiciaires spécifiques pose des problèmes essentiellement aux magistrats qui ne peuvent s'appuyer sur leurs ressources personnelles tels que leur expérience, leur reconnaissance institutionnelle et leur réseau professionnel. Les ressources personnelles constituent un élément fondamental pour saisir la manière dont les membres nationaux d'Eurojust peuvent affirmer leur autorité malgré des pouvoirs et des moyens limités et asymétriques.

La question du mode de désignation offre une première clef de compréhension intéressante afin de définir les ressources personnelles dont bénéficient chacun des membres nationaux. Chaque autorité centrale décide des critères de nomination de son représentant au collège. Affirmant la dimension accusatoire de ce dispositif, la seule obligation juridique est que tous ces magistrats soient des éléments du parquet ou des services du procureur. Une étude systématique des curriculum vitae des membres nationaux montre que la plupart des personnes nommées ont derrière elles une longue carrière judiciaire. En moyenne, on constate plus de quinze ans d'expérience généralement en tant que procureur au sein des autorités nationales. L'autre point commun réside dans une certaine spécialisation en matière de coopération judiciaire et/ou de lutte contre la «criminalité organisée ». Largement valorisée

\footnotetext{
${ }^{22}$ Entretien réalisé avec un magistrat d'Eurojust, mars 2004.

${ }^{23}$ Entretien réalisé avec un magistrat d'Eurojust, février 2006.
} 
dans les rapports officiels présentant chaque membre, l'ancienneté et la spécialisation ne peuvent cependant suffire à rendre homogène un tel groupe. En effet, si certains juges ont eu accès à des postes importants de direction à l'échelle de juridictions et de ministères, d'autres n’ont jamais été à ce niveau hiérarchique. Par ailleurs, il semble que la diversité des infractions regroupées sous le terme de «criminalité organisée» conduisent à des spécialisations très diverses (affaires de stupéfiants, criminalité financière, réseaux clandestins) ce qui n'est pas sans conséquence sur la manière dont les magistrats vont coopérer entre eux ou avec les autres autorités compétentes.

Dans ce contexte, derrière les règles juridiques et les discours officiels affirmant la collégialité du fonctionnement, certains témoignages tendent à montrer qu'il existe également une asymétrie quant aux ressources personnelles des membres d'Eurojust: «c'est vrai que durant les réunions du collège, on entend plus certains représentants que d'autres. Les prises de paroles n'ont pas la même valeur en fonction du magistrat ${ }^{24}$. Si cette variabilité des ressources personnelles a des effets sur le fonctionnement interne de l'unité, elle a également une importance dans la manière dont l'office judiciaire va développer son travail de coopération.

En effet, concernant la collaboration d'Eurojust avec les autorités judiciaires nationales, cette dimension se traduit par une différence de reconnaissance en fonction des pays : «le choix pour certains Etats de mettre à Eurojust des juges connus et reconnus permet à l'institution une meilleure visibilité à l'échelle nationale. A l'inverse, le choix pour d'autres de nommer des juges moins expérimentés représente une limite à notre action $»^{25}$. Dans cette logique, l'accumulation d'une longue expérience judicaire et de réseaux personnels multiples sont des voies de recours privilégiées pour les juges en quête d'affaires : "il est évident que quand vous connaissez directement les personnes du niveau national, parce que vous avez déjà travaillé ensemble, cela facilite les échanges. Il est nécessaire d'avoir une connaissance, et une expérience du terrain pour être efficace à Eurojust ${ }^{26}$.

Les ressources personnelles jouent, également, un rôle dans la manière dont collabore réellement Eurojust avec les autres unités européennes. En effet, en l'absence de règles juridiques de coopération contraignantes, les relations personnelles entre les membres de ces unités peuvent peser sur les logiques de collaborations.

\footnotetext{
${ }^{24}$ Entretien réalisé avec un magistrat d'Eurojust, mars 2004

${ }^{25}$ Entretien réalisé avec un magistrat d'Eurojust, juin 2005.

${ }^{26}$ Entretien réalisé avec un magistrat français, mai 2004.
} 
Dans le cas d'Europol, l'existence d'un roulement régulier au sein des bureaux de liaison, semble limiter la construction de relations de confiance entre les représentants nationaux de chaque office. La dimension professionnelle occupe aussi une place importante dans la façon dont les magistrats et les policiers de ces deux unités coopèrent. Selon un policier d'Europol: «en fonction des pays nous n'avons pas les mêmes relations avec les magistrats. Les magistrats italiens ou néerlandais sont souvent autoritaires avec les policiers de leurs pays. Mais cela ne passe pas avec les autres policiers européens $»^{27}$. Le fait quelques membres du collège d'Eurojust soient des officiers de police, en raison de l'hétérogénéité des systèmes judiciaires nationaux, permet dans certains cas de faciliter l'établissement de relation de travail avec les autorités policières. Concernant les rapports avec l'OLAF, il semble que les rares collaborations qui existent sont dues en grande partie aux relations personnelles des membres de chaque institution. En raison de leurs parcours professionnels, certains représentants de l'OLAF et du collège d'Eurojust se sont croisés dans le cadre d'affaires nationales ou de réunions d'experts.

En raison de pouvoirs limités et asymétriques, les ressources personnelles représentent les principaux moyens pour les membres nationaux d'Eurojust de réussir à devenir des interlocuteurs légitimes de la coopération judiciaire. La variabilité des ressources au sein du collège conduit cependant à renforcer l'incertitude de son activité.

\subsection{La retraduction d'un champ de compétence flexible}

Les magistrats d'Eurojust vont largement utiliser la flexibilité de leur champ de compétence afin de tenter de s'imposer sur des contentieux aussi sensibles et légitimants que la lutte contre la criminalité financière, le terrorisme ou le recueil d'informations.

La décision-cadre du 28 février 2002 portant création d'Eurojust, stipule que la mission confiée à cette unité est de lutter «contre les formes graves de criminalité qui sont souvent le fait d'organisations transnationales » (Décision instituant Eurojust, 2002, article 3). Quelques lignes plus loin, l'expression «formes graves de criminalité organisée » est employée afin de définir les infractions sur lesquelles la nouvelle unité judiciaire européenne peut intervenir. Le champ de compétence qui se construit via l'utilisation de ces termes peut au premier abord paraître réduit. En réalité, la prise en compte des phénomènes criminels comme enjeu politique à l'échelle européenne s'est imposée, depuis le début des années 1980, autour d'une labellisation politique prenant en compte un ensemble toujours plus large d'infractions sous ce terme (Bigo, 1996, p 249 ; Den Boer, 2002). Dans ces conditions,

\footnotetext{
${ }^{27}$ Entretien réalisé avec un membre d'Europol, février 2006.
} 
l'utilisation du concept de « criminalité organisée » apparaît comme une ressource importante en tant que catégorie d'analyse fluctuante se nourrissant d'éléments politiques et stratégiques.

Sur le plan juridique, Mireille Delmas Marty souligne que : «l'expression d'organisation criminelle renvoie de façon quelque peu circulaire à 'l'organisation' d'infractions considérées comme 'graves', soit en raison de la peine encourue, soit par référence à une liste d'infractions visant les questions sensibles » (Delmas-Marty, 2004, p 283). Cette labellisation, qui imprègne l'ensemble des textes européens, conduit à la construction d'inventaires référençant des formes de criminalités hétérogènes. Dans le traité d'Amsterdam en 1997, le Conseil européen parle de lutter contre «la criminalité organisée ou autre, et notamment le terrorisme, la traite d'êtres humains et les crimes contre des enfants, le trafic de drogue, le trafic d'armes, la corruption et la fraude » (Traité d'Amsterdam, 1997). Ce type de classification se retrouve dans la grande majorité des décisions ou conventions européennes et notamment dans les conclusions de Tampere et de Laeken qui décident de la création d'Eurojust. C'est dans cette logique que s'inscrit l'article définissant son champ de compétence. L'unité est ainsi compétente pour :

«a) les types de criminalité et les infractions pour lesquels Europol a compétence (...)

b) et les types de criminalité suivants :

- la criminalité informatique

- la fraude et la corruption, ainsi que toute infraction pénale touchant aux intérêts financiers de la Communauté européenne

- le blanchiment des produits du crime

- la criminalité au détriment de l'environnement

- la participation à une organisation criminelle au sens de l'action commune 98/733/JAI du Conseil du 21 décembre 1998 relative à l'incrimination de la participation à une organisation criminelle dans les Etats membres de l'UE (...)» (Décision instituant Eurojust, 2002, article 4).

Le choix d'une telle liste positive doit également se comprendre par la volonté de la part des autorités nationales d'exploiter une classification déjà négociée et employée pour d'autres instruments, en l'espèce Europol. La combinaison de ces logiques aboutit à la mise en place d'un champ de compétence large qui répond avant tout pour les acteurs judiciaires à un souci d'opérationnalité. Comme le souligne un magistrat ayant participé aux négociations européennes, "l'intérêt est de construire un cadre légal le plus large possible, dans l'esprit 
d'une flexibilité maximale, qui conduit avant tout à rechercher l'efficacité ${ }^{28}$. Cette priorité est largement mise en avant par les magistrats d'Eurojust : "parler de formes graves de criminalité organisée nous permet d'intervenir sur de nombreux types d'infractions afin d'être le plus opérationnel possible ${ }^{29}$.

La volonté de l'unité de se positionner dans l'espace européen de sécurité comme un office à part entière influence directement la façon dont ce champ d'intervention va être retraduit de manière collective et individuelle au sein d'Eurojust : " il est bien sûr évident, que certaines affaires, certains domaines nous apparaissent plus importants que d'autres, à la fois pour leur conséquences judiciaires mais aussi politiques et stratégiques ${ }^{30}$. Le décalage entre la présentation officielle de l'unité et la réalité des dossiers traités est un exemple d'un tel ajustement.

A l'origine, comme le souligne l'un de ses membres : «La dimension multilatérale caractérise l'ensemble des affaires que nous devons traiter. Sur ce point nous avons une vraie plus-value par rapport aux magistrats de liaison ou au RJE qui s'occupe surtout d'affaires bilatérales $\gg{ }^{31}$. Néanmoins, si Eurojust a pour vocation première d'intervenir sur les affaires mettant en cause plus de deux Etats, la réalité opérationnelle apparait différente : «ce n'est pas possible d'intervenir uniquement sur les affaires qui concernent au moins trois Etats. On aurait pas beaucoup d'affaires si l'on prenait que ces dossiers ${ }^{32}$. En 2002, il a été recensé 144 cas bilatéraux pour 58 cas multilatéraux et en 2003, 222 affaires bilatérales pour 78 affaires multilatérales. Cette tendance se confirme dans les statistiques pour les années 2004 et 2005. Eurojust oriente donc son activité, par nécessité et par choix, sur les affaires bilatérales qui pourtant ne représentent pas le cœur de son domaine de compétence, mais plutôt celui d'autres dispositifs d'entraide pénale tels que les magistrats de liaison ou le Réseau judiciaire européen.

L'étude des statistiques relatives aux délits traités par Eurojust offre également un point de vue intéressant. Ces statistiques prennent en compte les dossiers enregistrés et les classent sous différentes catégories. Si la construction de telles catégories répond à une volonté de définir la nature des infractions, leur pertinence reste relative et incertaine face à l’hétérogénéité de ces dernières.

\footnotetext{
${ }^{28}$ Ibid.

${ }^{29}$ Entretien réalisé avec un magistrat d'Eurojust, février 2006.

${ }^{30}$ Entretien réalisé avec un magistrat d'Eurojust, mars 2004.

${ }^{31}$ Entretien réalisé avec un magistrat d'Eurojust, février 2003.

${ }^{32}$ Ibid.
} 
Depuis sa création en 2002, les affaires de stupéfiants et de fraudes représentent la majorité des dossiers traités, soit globalement $20 \%$ pour chaque catégorie. Cependant, si l'on prend les différentes classifications utilisées dans les données de 2005, on constate qu'il existe 4 voire 5 catégories qui peuvent être mises en étroite relation : escroquerie et fraude; délit contre la propriété ou les biens publics, y compris la fraude ; blanchiment d'argent ; fraude fiscale; fraude à la TVA. En regroupant l'ensemble de ces affaires financières, on obtient une écrasante majorité des dossiers de l'unité. Dés 2002, le collège d'Eurojust relève cette situation et estime qu'elle « serait en partie imputable à l'incidence élevée d'enquêtes et de poursuites concernant les fraudes à caractère transfrontalier mais aussi à des définitions et concepts différents de la notion de fraude d'une juridiction d'un État membre à l'autre » (Rapport Eurojust, 2002, p 10). Derrière ces premières explications, le choix des catégories statistiques n'étant pas neutre (Robert, 1985), il est essentiel de comprendre la façon dont Eurojust traite et classifie ses affaires à travers les relations de concurrence qu'entretient l'unité avec les autres offices européens.

Sur les affaires de fraudes financières, la mise en perspective avec le domaine de compétence de l'OLAF est clairement perçue par les membres nationaux d'Eurojust : «les affaires de criminalités financières sont importantes parce qu'elles sont présentes par essence dans beaucoup de dossiers criminels. Alors, c'est vrai que vis-à-vis de l'OLAF ce n'est pas toujours très simple $»^{33}$. Jusqu'en 2005, les affaires de fraudes aux intérêts financiers de la Communauté pris en charge par Eurojust n'apparaissent pas distinctement puisqu'elles sont regroupées dans la catégorie «autres affaires». Comme le souligne un magistrat: «il est évident qu'au début, il ne fallait pas trop montrer qu'Eurojust pouvait intervenir dans le champ de compétence de l'OLAF (...) Maintenant nous devons nous affirmer $»^{34}$. L'intervention dans ce domaine d'Eurojust apparaît comme un moyen pour ses membres de renforcer leur légitimité sur un terrain judiciaire où les projets de communautarisation des affaires pénales, en particulier via le projet Corpus Juris et son idée de Procureur européen, sont toujours d'actualités, puisque le projet de Constitution européenne contenait cette proposition.

L'usage du champ de compétence en tant que ressource institutionnelle prend également tout son sens dans l'exemple des affaires de terrorisme. Entrés en activité juste après les attentats du 11 septembre 2001, les magistrats d'Eurojust ont identifié le domaine du terrorisme comme un terrain important de légitimation. Dés 2002, le collège se positionne

\footnotetext{
${ }^{33}$ Entretien réalisé avec un magistrat français, mai 2006.

${ }^{34}$ Ibid.
} 
dans cette perspective : «La complexité de certains dossiers de terrorisme et l'importance de la création de réseaux pour lutter contre ce phénomène, pour lequel la collaboration d'Eurojust a été demandée, a fait du terrorisme une des priorités de l'unité » (Rapport Eurojust, 2002, p 10).

Largement renforcée depuis les attentats sur le sol européen de 2004 et de 2005, le positionnement de l'unité est de se présenter comme un interlocuteur pertinent sur ce terrain, notamment en tant que chaînon dans l'échange d'informations : "Eurojust peut mettre en relation des magistrats nationaux de différents pays afin qu'ils puissent discuter ensemble. Nous pouvons également servir de relais pour échanger des informations très précieuses ${ }^{35}$. Dans les faits, les affaires de terrorismes ne constituent qu'une faible part des dossiers enregistrés par Eurojust. En 2002, 9\% des dossiers traités concernaient le domaine du terrorisme, en $20036 \%$ (soit 18 affaires), en 2004 7\% (soit 33 affaires) et en 2005 5\% (soit 25 affaires). Comme le souligne un magistrat de l'unité : «sur le terrorisme, nous devons nous imposer car c'est un domaine stratégique. Mais le problème c'est que les magistrats antiterroristes ne sont pas très accueillants. En fait, tant qu'ils ne seront pas obligés de nous donner des informations, nous n'aurons que des miettes $\|^{36}$.

Les représentants d'Eurojust vont essayer d'investir cette problématique, que se soit au niveau institutionnel ou opérationnel. Lors des nombreuses conférences officielles concernant ce sujet, les magistrats de l'unité vont faire leur possible pour valoriser leur travail, dans une logique similaire à leur activité de sensibilisation vis-à-vis des juridictions nationales. Illustratif de cette volonté, la demande appuyée d'un membre national afin de participer, au côté des représentants des autres dispositifs antiterroristes européens, à une conférence devant les services policiers et judiciaires des Etats membres. C'est dans cette même dynamique, que s'inscrivent les réunions stratégiques impulsées par Eurojust sur les affaires de terrorisme. Largement valorisées par l'unité, ces réunions ont pour objectif de «montrer concrètement aux autorités nationales que nous avons des moyens importants pour les aider $»^{37}$. Cependant, le compte-rendu succinct de la réunion de mars 2005 sur cette question est assez révélateur de la place actuelle d'Eurojust en matière de coopération antiterroriste : « $L a$ situation ne s'est pas beaucoup améliorée. Seuls quelques Etats échangent, de façon régulière et structurée, des informations sur les activités de terrorisme avec Eurojust ; d'autre le font,

\footnotetext{
${ }^{35}$ Entretien réalisé avec un magistrat d'Eurojust, février 2006.

${ }^{36}$ Entretien réalisé avec un magistrat d'Eurojust, mars 2004.

${ }^{37}$ Entretien réalisé avec un magistrat d'Eurojust, février 2006.
} 
mais de façon non structurée. Mais pour la majorité des Etats, cet échange n'est que ponctuel, voir inexistant » (Rapport Eurojust, 2005, p 36).

Si sur le plan opérationnel, c'est-à-dire des affaires traités, cet investissement n'a donc que peu de répercussion (entre 2004 et 2005, le pourcentage d'affaires de terrorisme a même baissé de 7\% à 5\%), au niveau institutionnel, Eurojust réussit peu à peu à s'imposer à travers un élargissement de son champ de compétences et de ses prérogatives officielles. Dans la décision du 20 septembre 2005, le Conseil européen décide qu'à partir de juin 2006, les informations intéressant la lutte contre le terrorisme transfrontalier devront systématiquement être adressées à Europol et pour certaines à Eurojust (Décision du Conseil européen relative à l'échange d'informations et à la coopération concernant les infractions terroristes, 20 septembre 2005). Même si le rôle d'Eurojust reste largement secondaire en particulier vis-àvis d'Europol, cette inscription dans les textes officiels permet à Eurojust de mettre les autorités nationales devant leurs obligations : «Nous invitons tous les Etats membres à veiller à ce que les correspondants qu'ils désignent travaillent en coopération plus étroite avec Eurojust et lui fournissent des informations sur les activités terroristes, conformément à l'engagement pris par les ministres dans la décision du Conseil du 20 septembre $2005 »$ (Rapport Eurojust, 2005, p 99).

Enfin, Eurojust semble parallèlement vouloir se positionner sur le terrain du recueil de données judiciaires. Le contrôle de l'information constitue sans aucun doute l'un des principaux enjeux pour l'office judiciaire. Comme le souligne un membre national : «cela nous permettrait de montrer que nous pouvons être utiles, et entraînerait de facto les autorités nationales à collaborer avec nous ${ }^{38}$. Face à une unité Europol qui n'a eu de cesse d'accroître son rôle en matière de recueil d'informations et de mise en place de fichiers, la stratégie d'Eurojust s'inscrit dans un certain mimétisme qui doit se comprendre par l'importance donnée actuellement à la problématique de l'information dans le champ européen de la sécurite ${ }^{39}$. A travers l'adoption par le Conseil, le 24 février 2005, de règles de procédures concernant le traitement et la protection des données à caractère personnel, Eurojust a ainsi mis en place un système automatisé de gestion de dossiers (Case Management system - CMS). Le développement de ce dernier a pour finalité l'échange sécurisé d'informations judiciaires entre les membres d'Eurojust et les autorités nationales judiciaires.

\footnotetext{
${ }^{38}$ Ibid.

${ }^{39}$ Voir les analyses effectuées dans le cadre du programme européen Challenge $:$ http://www.libertysecurity.org
} 
Dans un souci de légitimer sa place dans l'espace européen de sécurité Eurojust va utiliser son champ de compétence afin de s'affirmer sur certaines problématiques en fonction de l'environnement et des enjeux politiques. De façon collective (à travers le collège) ou de manière individuelle, les membres nationaux cherchent à étendre et à renforcer leurs interventions sur des terrains qui leur semblent légitimant, du fait de l'orientation général du champ européen de la sécurité.

En raison d'une institutionnalisation incertaine, qui se traduit par un manque de collaboration effective et une forte instabilité juridique vis-à-vis des autorités nationales et des autres offices européens compétents, les membres d'Eurojust vont redéfinir, par leurs pratiques, le cadre formel de l'unité afin de pouvoir légitimer leur action. Cette quête de reconnaissance est une stratégie largement affirmée par les membres de l'unité que se soit au niveau du collège ou de leur action individuelle.

Dans ce contexte, l'organisation et les pouvoirs judiciaires asymétriques de l'unité, ainsi que sa forte dépendance vis-à-vis des autorités centrales de chaque Etats, constituent autant de contraintes pesant sur l'activité des membres nationaux. Les ressources personnelles représentent alors pour ces derniers un moyen important de s'extirper et de s'affranchir d'un tel environnement juridique et institutionnel. La réalité de la coopération européenne via Eurojust repose pour une part importante sur la capacité personnelle des magistrats de cette unité à instaurer des relations de confiance avec les juridictions nationales. Dès lors, en raison de la variabilité des pouvoirs judicaires qui sont donnés à chaque magistrat et des ressources que ces derniers peuvent mobiliser, l'intervention d'Eurojust se caractérise par un fort déséquilibre en fonction des pays. L'incertitude et la large place laissée à l'informel dans l'activité actuelle de cet office ne sont pas sans conséquence sur le degré de stabilité du régime pénal qui se met actuellement en place en Europe.

Enfin, la volonté d'Eurojust de s'affirmer comme un maillon essentiel des dispositifs de coopération, se traduit également par un usage stratégique de ses compétences. On constate un décalage entre l'activité réelle et le positionnement institutionnel en faveur de la prise en compte de certaines catégories d'infractions considérées comme légitimantes du fait des logiques qui structurent le champ européen de la sécurité. L'investissement croissant d'Eurojust dans le recueil d'informations, le terrorisme et les affaires financières, tend à multiplier les voies de coopération concurrentes sur ces sujets et donc à conforter la 
superposition des dispositifs en charge de la coopération européenne. Une telle évolution conduit finalement à renforcer la complexité et l'opacité de l'espace européen de sécurité sur le terrain opérationnel et normatif.

\section{Bibliographie :}

Bastard B., Mouhanna C., Ackermann W. (2005), Une justice dans l'urgence. Le traitement en temps réel des affaires pénales, Paris, Mission de recherche Droit et Justice.

Bigo D. (1996), Polices en réseaux : l'expérience européenne, Paris, Presses de Sciences Po.

Delmas-Marty M. (2004), Les Forces imaginantes du droit: Le relatif et l'universel, Paris, Le Seuil.

Delpeuch T., Vigour, C. (2004), «Création et changement institutionnels », Boussague L., Jacquot S., Ravinet P. dir., Dictionnaire des politiques publiques, Paris, Les Presses de Sciences Po, p. 137-145.

Den Boer M. (dir) (2002), Organised crime : a catalyst in the Europeanisation of national police and prosecution agencies?, Maastricht, European Institute of Public Administration.

Domenach J. (2006), «Le droit de l'espace judiciaire pénal européen : un nouveau modèle juridique », Cultures \& conflits, Paris, l'Harmattan, $\mathrm{n}^{\circ} 62, \mathrm{p}$ 149-168.

Mangenot M., Michel H., Paris, N. (2005), Une Europe de la justice : mobilisation contre la "criminalité organisée" et institutionnalisation d'un espace judiciaire pénal européen (19962001), Paris, Mission de recherche droit et justice.

Mégie A., Ravinet P. (2007), «Processus intergouvernementaux et européanisation: La construction des espaces européens de l'enseignement supérieur et de la justice », dans Palier B., Surel Y. et. al, L'Europe en action : analyses d'européanisation, Paris, l'Harmattan, p 87144.

Mégie A. (2006), «Généalogie de la coopération judiciaire », Cultures \& conflits, Paris, $1^{\prime}$ Harmattan, $n^{\circ} 62$, p 11-42.

Musselin C. (2005), « Deux approches pour un même objet? », Revue française de science politique, Paris, vol.55, $\mathrm{n}^{\circ} 1, \mathrm{p} .51-63$

Pujas V. (2006), « Les difficultés de l'OLAF pour s'imposer en tant qu'acteur légitime de la protection des intérêts économiques et financiers européens », Cultures \& conflits, Paris, 1'Harmattan, ${ }^{\circ} 62$, p 107-128.

Robert P. (1985), Les comptes du crime : les délinquances en France et leurs mesures, Paris, Sycomore. 


\section{Textes officiels}

Conseil de l'Union européenne (1999), Conclusions de la présidence, Tampere, 15 et 16 octobre.

Rapports annuels de l'unité Eurojust (2002-2005), disponible à l'adresse : http://www.eurojust.europa.eu/press_annual.htm

Conseil de 1'Union européenne (2002), Décision 187/JAI instituant Eurojust afin de renforcer la lutte contre les formes graves de criminalité, JO C 063 du 06/03/2002.

Conseil de l'Union européenne (2005), Décision 671/JAI relative à l'échange d'informations et à la coopération concernant les infractions terroristes, JO C 253 du 29/09/2005.

Traité d'Amsterdam modifiant les traités sur l'Union européenne, JO C 340 du 10/11/1997.

Rapport d'information de Pierre BRANA (2002), délégation pour l'Union européenne de l'Assemblée nationale, $\mathrm{n}^{\circ} 3609$.

Eurojust et Europol (2004), Agreement between Eurojust and Europol, disponible à l'adresse : http://www.europol.europa.eu/legal/agreements/Agreements/17374.pdf 